\title{
EDITORIAL
}

\section{Clinical stability and switch therapy in hospitalised patients with community-acquired pneumonia: are we} there yet?

\author{
Julio A. Ramirez
}

I $\mathrm{n}$ this issue of the European Respiratory Journal, ENGEL et al. [1] studied barriers for early switch therapy in hospitalised patients with community-acquired pneumonia (CAP). The authors reference our initial work in the field of switch therapy, published in 1995 in Archives of Internal Medicine [2]. In hospitalised patients with CAP, the standard of practice before 1995 was to use intravenous antibiotics for the full duration of therapy. During our initial study, we obtained consent from patients to see if they were willing to participate in a trial using oral antibiotics once they reached clinical stability. Our Institutional Review Board requested a full evaluation of the initial 10 patients enrolled in the trial to be sure that a switch to oral antibiotics would not be associated with poor clinical outcomes. During the development of the protocol we decided to define clinical stability using two symptoms of CAP (cough and sputum production) and two signs of systemic response (fever and leukocytosis). Patients were defined as clinically stable when cough and sputum production were improved from the prior day, fever was resolved for at least $8 \mathrm{~h}$ and leukocytosis improved from the prior day by at least $10 \%$. Patients were considered candidates for switch therapy once they reached clinical stability and could tolerate oral intake. The determination of the patient being afebrile for at least $8 \mathrm{~h}$ was based on two factors: 1) if an antipyretic, such as acetaminophen, was used (duration of action of 4-6 h) and the patient was still febrile, an elevated temperature will be evident in $8 \mathrm{~h}$; and 2) nurses in the medical wards at the Louisville Veterans Hospital (Louisville, KY, USA) obtain vital signs every $8 \mathrm{~h}$. Our initial selection of criteria to define "clinical stability" in hospitalised patients with CAP was clearly a combination of science and feasibility. We recognised that for a new approach to be accepted in clinical practice it needed to be easy to implement.

In 1999, the American Thoracic Society (ATS) invited a group of clinical investigators to develop the first society guidelines for the management of CAP. At that time, I presented data on switch therapy generated by our group and other investigators. The committee determined that switch therapy was well studied and should be recommended as part of standard

Division of Infectious Diseases, University of Louisville, Louisville, KY, USA

CORRESPONDENCE: J.A. Ramirez, Division of Infectious Diseases, University of Louisville, Suite 104, 627 South Preston St, Louisville, KY 40202, USA. E-mail: j.ramirez@louisville.edu management of hospitalised patients with CAP. With a committee composed of investigators from the USA, Canada, Spain and the UK, the ATS 2001 guidelines can be considered the first international guidelines recommending switch therapy in the management of CAP [3]. The same year that the ATS guidelines were published, the first meta-analysis on the topic of switch therapy was published [4]. Several publications indicated that switch therapy was clinically effective after a patient reached clinical stability, even though investigators have used different criteria to define when a patient with CAP has reached clinical stability.

Returning to the question posed in this editorial: Are we there yet? The answer is clearly "no". Although the switch therapy approach was initially published more than 15 yrs ago and recommended by pneumonia guidelines more than 10 yrs ago, today it is used in only half of hospitalised patients with CAP. We are not there yet because we have not been able to translate the switch therapy approach from clinical research to clinical practice.

Biomedical research can be defined as the generation of new knowledge with the goal to improve patient care. It has been recognised that a significant proportion of the new knowledge generated by biomedical research does not translate into better outcomes for our patients. The National Institute of Health recognises two primary areas where new knowledge is not properly translated [5]. A gap exists in the translation of knowledge from basic research to clinical research. Defined as a T1 translation, it is due primarily to a lack of communication between basic and clinical investigators. A gap also exists in the translation of knowledge from clinical research to actual clinical practice. Defined as a T2 translation, it is due primarily to the lack of investigators performing implementation research (fig. 1). Without translating new knowledge from clinical research to clinical practice, our patients will not benefit from our work as investigators.

Figure 1 represents the new research approach suggested by the National Institutes of Health, the clinical and translational research model, with flow of knowledge and necessary interaction among investigators leading to the ultimate goal of improving patient outcomes. The study by ENGEL et al. [1] in this issue is a clear example of implementation research. The authors are generating new knowledge with the goal to move the switch therapy approach from clinical research to clinical 


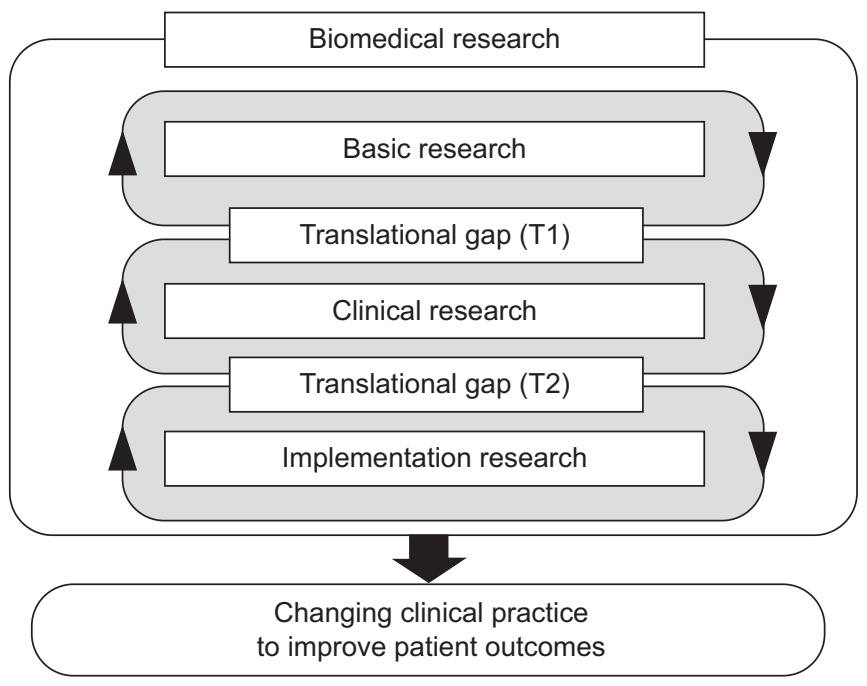

FIGURE 1. The clinical and translational research model.

practice. Switch therapy is just one of several approaches in respiratory medicine that is waiting to be translated to clinical practice. The fact that the editors of this journal are willing to publish a manuscript on switch therapy implementation should encourage investigators to become involved in implementation research in other areas of pulmonary medicine.

\section{STATEMENT OF INTEREST}

None declared.

\section{REFERENCES}

1 Engel MF, Postma DF, Hulscher MEJL, et al. Barriers to an early switch from intravenous to oral antibiotic therapy in hospitalised patients with CAP. Eur Respir J 2013; 41: 123-130.

2 Ramirez JA, Srinath L, Ahkee S, et al. Early switch from intravenous to oral cephalosporins in the treatment of hospitalized patients with community-acquired pneumonia. Arch Intern Med 1995; 155: 1273-1276.

3 Niederman MS, Mandell LA, Anzueto A, et al. Guidelines for the management of adults with community-acquired pneumonia: diagnosis, assessment of severity, antimicrobial therapy, and prevention. Am J Respir Crit Care Med 2001; 163: 1730-1754.

4 Rhew DC, Tu GS, Ofman J, et al. Early switch and early discharge strategies in patients with community-acquired pneumonia: a metaanalysis. Arch Intern Med 2001; 161: 722-727.

5 Woolf $\mathrm{SH}$. The meaning of translational research and why it matters. JAMA 2008; 299: 211-213. 